\section{DIFICULTADES CON PHYSICA II 4: LA CONFRONTACIÓN DIALÉCTICA DE ARISTÓTELES CON LAS OPINIONES PRECEDENTES SOBRE EL AZAR Y LA DISPUTA EN TORNO A LA IDENTIDAD DE SUS PORTAVOCES}

\author{
Iván de los Ríos Gutiérrez \\ Universidad Andrés Bello (Chile) \\ i.delosrios@unab.cl \\ ivandelosrios@yahoo.es
}

\section{DIFFICULTIES WITH PHYSICA II 4: THE ARISTOTELIAN DIALECTICAL CONFRONTATION WITH OPINIONS ON CHANCE AND THE DEBATE ON THE IDENTITY OF THE SPOKESPERSONS}

Cómo citar este artículo/Citation: Ríos Gutiérrez, I. de los (2014). "Dificultades con Physica II 4: la confrontación dialéctica de Aristóteles con las opiniones precedentes sobre el azar y la disputa en torno a la identidad de sus portavoces". Arbor, 190 (769): a163. doi: http://dx.doi.org/10.3989/ arbor.2014.769n5002

Recibido: 13 febrero 2014. Aceptado: 18 abril 2014.

RESUMEN: En este artículo se examina la exposición crítica de los tres grupos de opiniones sobre el azar que Aristóteles presenta en Physica [Phys.] II 4 y se pregunta por la identidad de sus portavoces. Aristóteles confronta tres creencias establecidas de acuerdo con las cuales: i) nada sucede por azar; ii) el azar es el origen de todas las cosas; iii) el azar es el instrumento un orden divino inescrutable al razonamiento humano. No obstante, en ningún momento encontramos referencias explícitas a uno o varios pensadores. ¿Quiénes son los negadores del azar y quiénes sus defensores? ¿A quién pertenece la identificación del azar con funciones divinas o divinidades singulares? Objetivo del presente artículo es perfilar el debate en torno a identidad de los defensores de las tres posiciones y contribuir, en la medida de lo posible, a su esclarecimiento en el marco de la crítica aristotélica del atomismo antiguo.
Copyright: (c) 2014 CSIC. Este es un artículo de acceso abierto distribuido bajo los términos de la licencia Creative Commons Attribution-Non Commercial (by-nc) Spain 3.0.
ABSTRACT: This article examines Aristotle's critical account of the three groups of opinions on chance presented in Physica [Phys.] II 4-6 and considers the question of the identity of the advocates of these ideas. Aristotle confronts three common beliefs according to which: i) nothing happens by chance; ii) chance is the origin of everything; iii) chance is the instrument of a divine dimension inscrutable to the human mind. However, we find no explicit references to any authors. Who are the deniers of chance and who are its proponents? Who identifies chance with divine functions or particular deities? This article aims to outline the debate on the identity of the advocates of these common beliefs and to contribute to its clarification within the frame of the Aristotelian criticism on ancient atomism. 


\section{INTRODUCCIÓN}

A lo largo del capítulo 4 del segundo libro de la Physica, Aristóteles elabora un ensayo de confrontación dialéctica con las opiniones precedentes en torno a túxn y aútó $\mu \alpha \tau o v$. Lejos de verse reducidas al terreno exclusivo de la prosa especulativa, dichas opiniones portan el sentido que la lengua del estagirita atribuye a expresiones de uso corriente según las cuales algunos acontecimientos se pro-

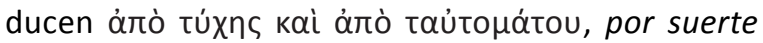
y por causalidad ${ }^{1}$. Los comentaristas antiguos otorgan una importancia capital al plano del lenguaje cotidiano como ámbito prologal de la investigación aristotélica sobre el azar. Temistio, Simplicio y Juan Filópono coinciden en subrayar que la doctrina del maestro tiene su punto de partida en el lenguaje cotidiano y que, en este sentido, la intención de Aristóteles no es otra que la de analizar el significado de aquello que los hombres presuponen cuando hablan de rúxn y aútópatov como causas de sucesos fortuitos ${ }^{2}$. Sin embargo, el examen del vocabulario del azar no basta para la investigación del ser natural en cuyo interior encontramos la primera doctrina filosófica sobre la suerte y la casualidad. Tomando como punto de referencia los usos lingüísticos, Aristóteles constata la existencia de una serie de expresiones que remiten a sucesos atribuidos a

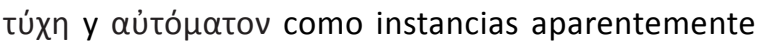
causales: "Se suele decir también que son causas la suerte y la casualidad y que muchas cosas son y acontecen debido a la suerte y a la casualidad..." ${ }^{3}$. Dado que tales son las cosas que se dicen ( $\lambda \varepsilon \dot{\gamma \varepsilon \tau \alpha \iota)}$ en torno al azar, parece pertinente realizar un análisis pormenorizado del lenguaje corriente katà tr̀v

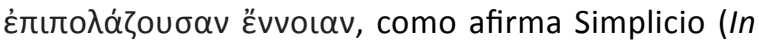
Phys. 357, 4), i.e., según la idea predominante de que algunos acontecimientos se deben a la suerte y la casualidad. Se trata de un análisis que combine la creencia común filtrada en el lenguaje con las enseñanzas de los filósofos precedentes que se han pronunciado al respecto. Dicha combinación constituye la base sobre la que Aristóteles elabora su propuesta sobre el azar en Phys. II 5-6 y, en este sentido, representa una primera confrontación filosófica con opiniones históricamente transmitidas por la tradición ${ }^{4}$. Objetivo del presente artículo será perfilar las líneas principales de dicha confrontación y tratar de dilucidar, desde el interior de una vieja polémica aún vigente, quiénes son los interlocutores de Aristóteles en su revisión crítica de las opiniones precedentes en torno a la existencia y naturaleza de

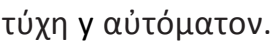

1.


aútónatov como causas de cierto tipo de acontecimientos conduce a Aristóteles a una primera declaración de intenciones en Phys. 195b31-33 (Cf. II 4 196b7ss.): "Se suele decir también que son causas la


chas cosas son y acontecen debido a la suerte y a la casualidad. Hay que examinar, entonces: a) de qué manera la suerte y la casualidad se encuentran entre las causas que hemos indicado; b) si la suerte y la casualidad son lo mismo o son diferentes, y, c) en general, qué es lo que son" ${ }^{5}$. Nótese que las razones que impulsan a una primera formulación de objetivos no son otras que la costumbre, el uso y el hábito lingüístico que conduce a las gentes y a los más sabios a atribuir

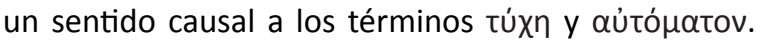
Siendo tal el caso, y dado que Aristóteles acaba de establecer en Phys. II 3 su doctrina etiológica, parece necesario preguntar por la posibilidad de incluir aquello que todos identifican con una cierta causa en la clasificación previamente esbozada: ¿cómo localizar a túxn y aútó $\mu \alpha \tau o v$ entre las causas descritas por Aristóteles en su investigación del ser natural? ${ }^{6}$ ¿Existe alguna diferencia entre ambas $y$, en caso afirmativo, en qué consiste? ¿Qué son, en definitiva, túxך y aútó $\mu \alpha \tau o v$ ? Aristóteles responderá más tarde a estas preguntas $^{7}$. Por el momento, se limita a presentar de modo esquemático y ciertamente confuso tres grupos de opiniones fundamentales en torno a la existencia y la naturaleza de la suerte y la casualidad:

1) En primer lugar, la de todos aquellos que, de un modo u otro, rechazan la existencia de túxn y

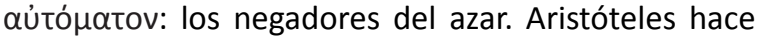
referencia a ellos en $195 \mathrm{~b} 36 \mathrm{ss}$ :

\footnotetext{
"Algunos (हैviol) dudan de su existencia y afirman que nada proviene de la suerte, sino que hay siempre una causa determinada de todo cuanto decimos que

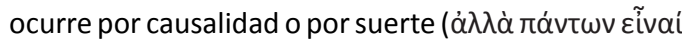

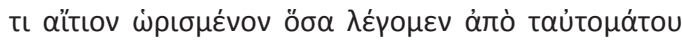

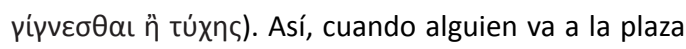
y encuentra fortuitamente a quien se deseaba pero que no se esperaba encontrar, ellos pretenden que la causa está en haber querido ir a la plaza por determinados asuntos. $Y$ de la misma manera en los otros casos que se atribuyen a la suerte: siempre es posible encontrarles una causa, y esta no es la suerte."
}

2) En segundo lugar, Aristóteles considera las propuestas de quienes atribuyen al azar y la espontaneidad el origen del cosmos, afirmando, por tanto, su 
existencia, pero restringiendo su ámbito de aplicación al plano cosmogónico. En efecto, a juicio de Aristóteles, tales autores defenderían simultáneamente la existencia del azar como principio absoluto de cosmogénesis y su completa ausencia en la dimensión sublunar de los procesos naturales. Este y todos los mundos posibles habrían sido producidos espontáneamente merced a un torbellino primigenio desde el que se organiza paulatinamente el orden de todas las cosas. Un orden natural que operaría según leyes estrictamente causales descartando, así, todo fenómeno genuinamente fortuito. De este modo, el azar queda identificado en 196a24-35 con una potencia cosmogónica cuya operatividad, sin embargo, es eliminada del ámbito sublunar:

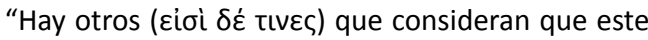
mundo y todos los mundos son productos de la casualidad; pues dicen que el torbellino surgió por ca-

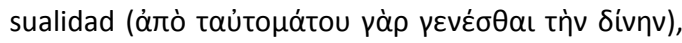
como también el movimiento que separó las partes y estableció el actual orden del Todo. Y esto es lo que más nos sorprende; pues dicen, por una parte, que los animales y las plantas no son ni se generan fortuitamente (árò túxns), sino que la causa es la naturaleza, o una Inteligencia, o alguna otra semejante (porque de una determinada semilla no se genera fortuitamente cualquier cosa, sino de esta semilla un olivo, de aquella un hombre), y dicen, por otra parte, que el cielo y las cosas más divinas que vemos se han generado por casualidad (árò toũ aủto hátou), y que sus causas no son las mismas que las que han generado a los animales y las plantas".
}

3) Por último, en 196b5-7 encontramos la opinión de quienes identifican el azar con cierto tipo de causa y admiten su existencia y operatividad, si bien subrayando su naturaleza insondable para el intelecto humano y, por tanto, favoreciendo una interpretación religiosa del acontecimiento fortuito:

"Hay también otros que piensan que la suerte es una causa, pero que es algo divino y tan demónico

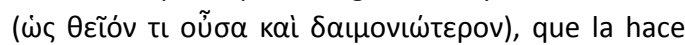
inescrutable al pensamiento humano". ${ }^{8}$

La exposición esquemática de las opiniones sobre el azar invita a formular varias preguntas: ¿quiénes son los negadores del azar y quiénes sus defensores? ¿A quién pertenece la identificación de la suerte y la casualidad con funciones divinas o divinidades singulares? ¿Cuál es la identidad de los हैvio en $195 \mathrm{~b} 36$ y de los tiveৎ en 196a24-26? Y lo que parece más importante: ¿hasta qué punto la tripartición aristotélica no constituye, como ha señalado Magris (1984, p. 437), un forzamiento de la realidad histórica? ${ }^{9}$ Frente a tales interrogantes, no podemos conformarnos con comentarios como los de William Charlton a Phys. II, quien sostiene que el capítulo 4 no presenta dificultad alguna (Charlton, 1970, p.105). De ser esto cierto, ¿a qué se debe el grado de confusión imperante entre los diversos especialistas? Volvamos por un instante a la exposición aristotélica de los tres grupos de opiniones. Aristóteles distingue un primer conjunto de creencias aceptadas y pensadores que coinciden en negar toda validez a los términos túxฤ y aútó $\mu \alpha \tau o v$ sobre la base de que no existe un acontecimiento al cual no pueda serle atribuida una causa concreta (Phys. II 4, 195b35-19 6a 25). La razón por la cual hablamos de sucesos fortuitos no puede deberse, por tanto, más que a la ignorancia humana y al indeterminismo psicológico derivado del desconocimiento de los mecanismos racionales férreamente establecidos que rigen el orden de la naturaleza. Una opinión que recuerda a los planteamientos elementales del atomismo antiguo. En este sentido, y sirviéndose de los comentarios de Simplicio y los estudios de Bailey (1922), David Ross aborda el problema de la identidad de los negadores del azar concluyendo que Aristóteles está pensando principalmente en Demócrito, "who, while he used chance in his кобнолоi $\alpha$, did not allow chance to be operative in details such as the finding of a treasure $^{\prime 10}$ (Ross, 1960, p. 514, ad loc. 195b36-19 6a 3). El fragmento 2 de Leucipo parece favorecer la hipótesis de Ross en la medida en que allí se enuncia una de las máximas fundamentales de la física atomista: "nada ocurre en vano, sino todo racionalmente y por necesidad" (Diels-Kranz, 1951-1952 [en adelante DK], 67 B

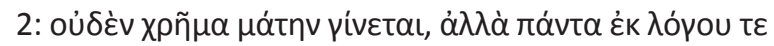

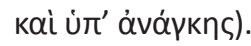

Si seguimos las indicaciones de Ross y acudimos a Simplicio, vemos que, en efecto, el comentarista bizantino lleva a cabo un esfuerzo considerable por aclarar la identidad de los adversarios del azar que, sin embargo, no le impide vacilaren sus conclusiones. En un primer acercamiento propone una confusa mezcolanza entre Anaxágoras, Demócrito y Empédocles, acompañados por la célebre frase de Polo citada por Aristóteles -el azar ama al arte y el arte al azar ${ }^{11}$ y la creencia popular en la túxn compartida por la religión tradicional (In. Phys. 327-328). La clave de toda la polémica se concentra en la expresión aristotélica

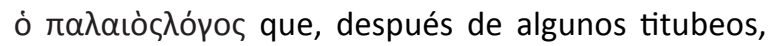
Simplicio termina atribuyendo a Demócrito (In. Phys. 330 14-20). Aristóteles está refutando el argumento de quienes suprimen por completo la suerte y la casualidad. Habiéndolo presentado en 195b36-196a13, 
el filósofo se dispone a desacreditar la idea de que un acontecimiento para el que es posible encontrar una causa no puede ser dicho "fortuito". Y es que, en efecto, aunque no ignoramos que, como decía el antiguo argumento que suprimía la suerte (ó $\pi \alpha \lambda \alpha$ เòs

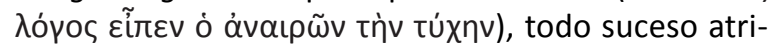
buido al azar puede ser remitido a una causa singular, lo cierto es que la opinión común y el lenguaje ordinario siguen distinguiendo entre lo que sucede fortuitamente y lo que no. ¿Qué significa aquí la fra-

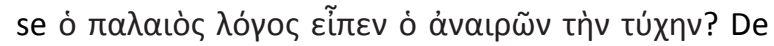
acuerdo con Ross (1960 ad loc.), la expresión puede tener dos significados distintos: "The words may mean (1) "the ancient argument", i.e., that put forward by the atomists (so S[implicio] 330.14), or (2) "the before-mentioned argument"12. Ross sostiene que el representante del primer grupo de opiniones es sin duda Demócrito. De hecho, cualquiera de los dos significados conduciría a un mismo destinatario. En el caso del "antiguo argumento", porque Ross ha sentenciado ya que se trata de "that put forward by the atomists" (Ross 1960 ibid.). En el caso anafórico del "argumento mencionado más arriba", porque el comentarista británico acaba de identificarlo con el pensamiento de Demócrito en el interior de su propia distinción. Sea como fuere, Ross opta por la primera de las dos posibilidades, esto es, por un sentido no

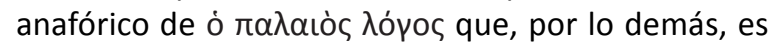
el que Aristóteles emplea en los otros lugares del corpus en los que hallamos expresiones similares ${ }^{13}$. Si retrocedemos hasta Simplicio, encontramos que la atribución del $\pi \alpha \lambda \alpha$ ı̀ç $\lambda o ́$ ó a Demócrito está apoyada, a su vez, en la supuesta opinión de Eudemo, quien, a juicio del comentarista, también refiere el argumento en cuestión al pensador atomista:

\footnotetext{
"La expresión "el antiguo argumento que suprime la suerte" parece referirse a Demócrito. Este, en efecto, pese a haber hecho uso de la suerte en su cosmogonía, en los casos particulares niega que la suerte pueda ser causa de nada, remitiendo siempre a otro tipo de causas. Por ejemplo, dice que la causa de encontrar un tesoro es cavar un hoyo o plantar un olivo, o que la causa de la fractura en el cráneo del hombre calvo es el hecho de que un águila dejara caer la tortuga para romper su caparazón. Esto es lo que cuenta Eudemo."14
}

Los ejemplos de Demócrito contribuyen a apoyar su candidatura como máximo representante de los negadores del azar ${ }^{15}$. De hecho, el relato del agricultor que encuentra casualmente un tesoro al plantar un árbol y el de la tortuga que rompe el cráneo de un viandante se prestan a la perfección al debate sobre el azar. No parecería, pues, existir dificultad alguna a la hora de desvelar la identidad del primer grupo de pensadores. Ahora bien: si asumimos esta hipótesis e identificamos a Demócrito y a sus discípulos con los negadores del azar, nos enfrentamos con un obstáculo evidente. $Y$ es que, en efecto, el segundo grupo de opiniones apunta también -y de manera indiscutible- a la escuela atomista, lo cual nos obligaría a admitir que Aristóteles está realizando una clasificación innecesaria, en la medida en que habla de un solo pensador o de un mismo grupo de pensadores. De hecho, Ross no duda en localizar al filósofo de Abdera también dentro del segundo grupo. Su confianza se apoya esta vez no solo en los testimonios de Simplicio (quien atribuye a Demócrito en 331.16 el argumento de 196a 24-35 - "sin duda acertadamente", afirma Ross ad loc.) sino, además, en los de Juan Filópono (In Phys, 261.31ss) ${ }^{16}$ :

\footnotetext{
“Pero hay algunos, dice [Aristóteles] refiriéndose a Demócrito y sus seguidores, que creyeron que era [el azar] la causa de este cielo y de las cosas más divinas entre lo manifiesto, pero no discutieron al respecto ni lo más mínimo".
}

¿Cómo es posible que un mismo filósofo pertenezca simultáneamente a los dos grupos? ¿Acaso Aristóteles entendía que Demócrito negaba y afirmaba al mismo tiempo la existencia del azar? ¿No es contradictorio que un autor que defiende el imperio de la necesidad en todo movimiento natural defienda, a la vez, el origen espontáneo del vórtice primordial? Tal vez la clave estaría en rechazar a Demócrito como autor del segundo grupo de opiniones y negar que atribuyera en su obra el origen del cosmos a un movimiento espontáneo, mecánico y fortuito. De hacerlo, entraríamos en un debate que incluye a autores como Cicerón, Lactancio o Tomás de Aquino, y que llega hasta el canto IV de la Divina Comedia, donde el poeta florentino afirma de Demócrito "che il mondo a caso pone"17. ¿Estaría Dante equivocado al sostener que Demócrito sitúa el azar en el origen del cosmos o, en realidad, el indeterminismo derivado de la teoría del vórtice debería ser interpretado en términos epistemológicos y psicológicos? ${ }^{18}$

Sea como fuere, el contenido del segundo grupo de opiniones se resume en la identificación del azar con una potencia cosmogónica que, sin embargo, carece de toda aplicabilidad en el seno de los procesos naturales. Demócrito, en efecto, parece haber defendido el origen fortuito del cosmos a partir de un torbellino que se pone en movimiento espontáneamente, dando lugar a la ordenación observable: 
"Del todo se segregó un torbellino de variadas

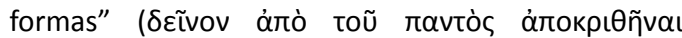

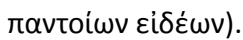

Se trata del fragmento DK 68 B 167, recogido por Simplicio en In Phys. 327, 24-2519. Unas líneas después (In Phys. 327, 26-27), Simplicio recuerda que es Eudemo quien atribuye esta concepción a Anaxágoras y a su creencia en un Intelecto que habría dispuesto ordenadamente todas las cosas partiendo de un movimiento espontáneo: "Y aunque Anaxágoras concede la existencia del Intelecto, como dice Eudemo, piensa que todas las cosas resultan del azar".20

A pesar de ello, el comentarista se decanta en un primer momento por Empédocles como autor de dichas teorías (DK 31 B 53, 75, 85, 98 y 103), para terminar remitiendo la doctrina definitivamente a los seguidores de Demócrito (In Phys. 331). ¿Quiénes son, pues, los pensadores incluidos por Aristóteles en el segundo grupo? Todas las opciones barajadas hasta el momento parecen factibles. Tanto Empédocles como Anaxágoras y Demócrito admiten un cierto margen de acción para el denominado azar en sus escritos. El primero, desde una consideración evolucionista de procesos naturales que avanzan por ensayo y error (y que puede rastrearse en DK 31 B 35, 53; DK 68 B 164, 167; cf. Phys. II 4, 196a11-24 y 198b27-b32, GC334a1ss y 333b7-16). Los segundos, en la medida en que reducen la validez del vocabulario del azar a las limitaciones del intelecto (DK 59 A 66; DK 68 B 3, 119, 176, 195, 269 y 286) $)^{21}$ y, a su vez, por cuanto eliminan toda interpretación teleológica del devenir natural. En efecto, el atomismo democríteo y el Nous anaxagórico parecen coincidir en la designación del origen espontáneo de todo orden cósmico, esto es, en la negación de un sentido teleológico inmanente que permee la complejidad de la naturaleza. Desde esta perspectiva, parece posible conjugar, como recuerdan Bailey (1922, pp. 139-143), y Ross (1960, p. 515), la creencia en el origen fortuito del cosmos con la defensa de un mecanicismo causal inflexible en el orden de los procesos empíricos. La necesidad de toda conexión causal convive en el materialismo jonio con la negación del carácter finalístico del devenir. Que el mundo haya sido configurado espontáneamente significa que, a pesar de estar sometido a una férrea organización causal necesaria en sus conexiones, no responde a designio trascendental, teleológico o providencial alguno. Por ello, tanto Empédocles como Anáxágoras y Demócrito parecen excelentes candidatos al segundo grupo de opiniones.
El tercer grupo es despachado por Aristóteles en apenas cinco o seis líneas de texto. A pesar de ello, la referencia al ámbito de la interpretación religiosa parece indiscutible y tal es la opinión generalizada de Temistio, Filópono y Simplicio en la Antigüedad, y de Ross, Boeri y Kaul en sus comentarios modernos a Phys. II 4.

2.

La confusión generada por Phys. II 4 en la literatura especializada no se debe tanto a Aristóteles como a sus modernos intérpretes. El estagirita se niega a ofrecer nombres propios a pesar de las evidentes conexiones entre los distintos grupos de opiniones. Muy probablemente, porque su interés se centra en la crítica generalizada del materialismo atomista y en el esclarecimiento de las contradicciones que se derivan de sus premisas, más que en el desenmascaramiento de autores individuales. No parece desmedido formular la hipótesis de que el anonimato y la intercambiabilidad que caracterizan las opiniones sobre el azar tuvieran una función específica en la mente del estagirita, a saber: mostrar la confusión reinante entre sus predecesores acerca de la existencia y la naturaleza de eso que denominamos acontecimientos fortuitos mediante los términos túxn y aútó $\mu \alpha \tau o v$. Más aún, subrayar que dicha confusión se debe, en buena medida, al hecho de que quienes se expresaron sobre la suerte y la casualidad lo hicieron de modo contradictorio, unificando en una sola doctrina aspectos conceptualmente irreconciliables, como en el caso de la tesis que afirma el origen fortuito del cosmos y la necesidad absoluta como rasgo esencial del movimiento natural. A juicio de Aristóteles, dicha confusión ha sido representada de manera explícita por el atomismo democríteo. En este sentido, cabe sostener que la motivación filosófica que pone en marcha la investigación aristotélica sobre el azar y, con ella, la confrontación dialéctica con la tradición, no es otra que el rechazo del materialismo necesitarista de la escuela atomista, que identifica la naturaleza con un plano de conexiones causales necesarias donde no hay margen de indeterminación objetiva, mientras que, simultáneamente, atribuye el orden del cosmos a un tipo de causa indeterminada. Frente a esta propuesta, y en el marco de unas investigaciones físicas gobernadas por la teleología ${ }^{22}$, Aristóteles define el azar en Phys. II 5 como una causa accidental en el orden de los fines de aquellas cosas que podrían haber sido producidas como efecto de la naturaleza o el pensamiento. Al hacerlo, fortalece la estructura teleológica de la naturaleza y la prioridad de la sustancia y del acto en la medida en que considera que todo 
suceso fortuito, casual e indeterminado presupone un marco estructural prioritario y fundante sobre el que emergen ocasionalmente excepciones significativas:... “...y puesto que nada accidental es anterior a lo que es por sí, es evidente que ninguna causa accidental es anterior a una causa por sí . La casualidad y la suerte son, entonces, posteriores a la inteligencia y la naturaleza. Así, incluso aunque se concediese que la casualidad es la causa del cielo, sería necesario que la inteligencia y la naturaleza fuesen antes causas no solo de muchas otras cosas, sino también de este Universo". Esta clara referencia a Demócrito y los atomistas cierra la doctrina del azar expuesta en Phys. II 4-6 (198a 5-15) ${ }^{23}$. En mi opinión, no es casual que Aristóteles cierre su investigación insistiendo en aquello que en un primer momento le llevó a criticar las propuestas de interpretación de lo fortuito vigentes en la tradición y, en concreto, aquellas que atribuyen a la casualidad el origen del cosmos. La investigación aristotélica del azar parte de un ensayo de refutación de toda teoría cosmológica, física y metafísica que, como la atomista, atribuya el plano de la más perfecta determinación natural a causas indeterminadas. La crítica de Aristóteles al atomismo y a su teoría del vórtice es comprensible, por tanto, en la medida en que su aceptación quebraría algunos presupuestos básicos del aristotelismo como la prioridad de la sustancia sobre el accidente en el plano estructural del ser, o la del acto sobre la potencia en el ámbito dinámico del devenir.

Si esto es cierto, Aristóteles no estaría principalmente interesado en los autores específicos que defienden tal o cual propuesta, sino en las direcciones posibles que ha de tomar toda interpretación filosófica del azar con pretensiones de verdad. Direcciones que, si bien presentes en el pensamiento y la cultura antiguos, no lo están sino de modo confuso, indiferenciado y balbuciente. Labor del investigador natural será, entonces, mostrar esos trazos, trabajar críticamente sobre ellos y extraer una propuesta teóricamente sólida partiendo de los mismos, tal y como Aristóteles se propone en Phys. II 5-6. Esto no quiere decir que hayamos de conformarnos con el anonimato de Phys. II 4, sino, más bien, que el aspecto central de dicho capítulo reside en sus contradicciones internas, tanto desde el punto de vista de la exposición como desde el contenido de las propuestas analizadas por el estagirita. Teniendo en cuenta estos aspectos, considero que el primer grupo de opiniones descrito en 195b36-196a24 recuerda inevitablemente al principio ilustrado del pensamiento científico y, en concreto, a la formulación anaxagórea y democrítea del azar que será retomada por la filosofía estoica:
“A.[Anaxágoras], Demócrito y los estoicos [afirman que el azar] es una causa oculta al entendimiento humano. Algunas cosas, en efecto, se producen por necesidad, otras según el destino, otras de acuerdo con la decisión, otras por suerte, otras por causalidad". ${ }^{24}$

Como es sabido, el naturalismo científico del pensamiento ilustrado coincide en designar como azar, suerte o casualidad a la imposibilidad epistémica de individuar causas específicas para fenómenos concretos. Ahora bien, a juicio de estos autores, dicha imposibilidad debe ser atribuida a los límites del intelecto humano, y no a la indeterminación de la naturaleza, que opera siempre y necesariamente según férreas conexiones causales. El azar no existe, por tanto, más que al modo de un testimonium paupertatis o merma intelectual que el progreso científico y el paulatino descubrimiento de las verdaderas causas de todo acontecer despejarán definitivamente $^{25}$. El imperio de la causalidad es, en definitiva, uno de los grandes logros del naturalismo científico ilustrado que parte de la filosofía de Anaxágoras y es radicalizado por el mecanicismo de Demócrito. Esta línea de interpretación de la complejidad ontológica y de los instrumentos de interpretación de la misma sería la que Aristóteles tiene en mente a la hora de designar un primer conjunto de opiniones sobre el azar.

En relación con el segundo grupo, los fragmentos conservados nos obligan a aceptar la hipótesis de Simplicio, Ross y Boeri, según la cual Demócrito pertenecería simultáneamente al primer y al segundo grupo de opiniones. Ello no obsta para señalar un grave error que el comentarista británico pasa por alto en su lectura de Simplicio. En efecto, cuando Simplicio rastrea

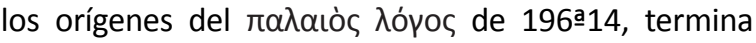
por remitirlo no ya al primer grupo de opiniones -los negadores del azar- sino al segundo, pues los pasajes de 330.16-17 en los que este es considerado remiten indudablemente a 196s24-196b5, es decir, al conjunto de autores que identifican el azar con una causa cosmogónica y lo hacen desaparecer del ámbito natural. Ross está en lo cierto cuando considera que el $\pi \alpha \lambda \alpha i o ̀$ $\lambda$ ójos viene referido al "argumento anteriormente citado", esto es, a los defendidos por los negadores del azar en 196a1-7. Si esto es así, la propia exposición aristotélica daría pie a múltiples conexiones y filtraciones entre los dos primeros grupos y, en este sentido, parece plausible aceptar que un mismo pensador pertenezca grupos distintos. Uno o varios pensadores que, como hemos señalado más arriba, están siendo juzgados por Aristóteles precisamente en la medida en que afirman cosas contradictorias, es decir, porque defienden principios excluyentes que, en cuanto tales, deberían ser 
incluidos en propuestas teóricas distintas. Anaxágoras, Empédocles o Demócrito resultan, en este sentido, claros representantes del segundo grupo de opiniones.

Por último, la opinión de quienes ven en túxn y aútó $\mu \alpha$ tov causas misteriosas vinculadas al orden de la trascendencia bien podría ser puesta en relación con el grupo de los negadores del azar descrito en 196a1-7. En efecto, ambas opiniones contienen implícita o explícita-

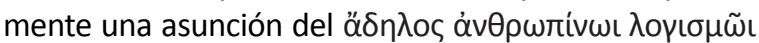
(causa oculta al razonamiento humano) anaxagórico y democríteo (Phys. 196b5-7), si bien en el caso de estos últimos la interpretación de la inescrutabilidad de lo ä $\delta \eta \lambda o \varsigma^{26}$ queda revestida de un tono religioso o, como afirma Aubenque (1963, p.84), de una "concepción mística del azar". Así lo entiende Simplicio en 333, 5-20, quien, entre otros candidatos a representar al tercer grupo de opiniones, menciona a los estoicos, el culto

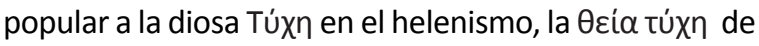
Platón, los himnos órficos e, incluso, uno de los oráculos délficos ${ }^{27}$. Aecio, por su parte, ya había atribuido la expresión å $\delta \eta \lambda o \varsigma ~ \alpha i t i \alpha$ a Anaxágoras y Demócrito (DK 59 A 66). Esta concepción del azar es, por lo demás, la que encontramos recogida en los Stoicorum Veterum Fragmenta de von Arnim, que reproducen, como vemos, casi a la perfección, la expresión utilizada por Aristóteles en

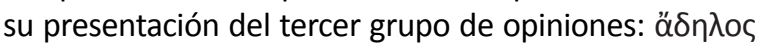

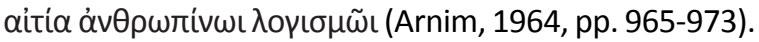
Por lo demás, es importante señalar que en este último ह̌vઠołov se condensa la riqueza, densidad y complejidad de toda la tradición religiosa y filosófica previa a Aristóteles. Una tradición que parte de los poemas homéricos y que, bajo cosmovisiones distintas y en momentos históricos diversos, interpreta el acontecimiento fortuito bien desde el punto de vista de la trascendencia, bien desde el de la inmanencia: de la trascendencia, en la medida en que lo fortuito se presenta como golpe del destino ( $\mu$ oĩpa),envío de los dioses o expresión de la voluntad de Zeus, i.e., en la medida en que la casualidad encubre y expresa el orden férreo de todo lo que existe y las limitaciones del intelecto humano para captarlo en su plenitud ya siempre significativa; de la inmanencia, por cuanto el azar, desprovisto de trasfondo teológico, pasa a ser interpretado en clave ilustrada como margen ineludible de indeterminación en un universo contingente abierto a la impredictibilidad del futuro y constituido por el entrecruzamiento de la naturaleza, la decisión y el azar. La experiencia humana de la felicidad y la desgracia, de la buena y la mala suerte, del acontecimiento significativo indescifrable ha exigido siempre de estrategias de comprensión que posibiliten un acceso epistémico al sentido de aquello que nos acontece de modo inesperado, accidental y eminentemente signifi- cativo. Aristóteles se hace cargo de este desafío filosófico en sus investigaciones naturales motivado por el deseo de dotar de inteligibilidad al acontecimiento fortuito sin, por ello, reducirlo al esquema arcaico y al impulso teológico que descubre en la casualidad el rostro oculto del dios o la implacable ejecución del destino. En este sentido, si bien la confrontación dialéctica de Aristóteles con la tradición en Phys. II 4 adolece de una notable falta de claridad, nos muestra hasta qué punto el filósofo forma parte de una corriente cultural que, en todas sus formas expresivas, quiso contribuir a la siempre imperfecta domesticación de aquello que, al decir de Píndaro en Olímpica XII, gobierna la existencia en todos sus ámbitos. En mi opinión, y complementando su desarrollo especulativo en Phys. II 5-6, la relevancia de la doctrina aristotélica del azar también reside en su contextualización en el marco de las fuentes que limitan, nutren y generan la prosa filosófica actuando como su horizonte de sentido, esto es, el marco de las distintas modalidades expresivas de la cultura griega tal y como nos han sido históricamente transmitidas en la épica homérica, la lírica arcaica y el drama ático, así como el espíritu crítico encarnado con posterioridad en la prosa científica de la Ilustración ateniense. La doctrina aristotélica del azar, si bien inaugural y filosóficamente autónoma, constituye un momento clave en la historia del pensamiento humano por cuanto tiene, precisamente, de pre-filosófica $y$ arcaizante ${ }^{28}$. El problema del azar que se viene anunciando en el vocablo griego túxn y, más aún, el vocablo mismo, configuran una instancia lingüística muy anterior a Aristóteles que condensa en su interior una determinada experiencia humana de la incertidumbre como rasgo esencial de la existencia humana. Desde este punto de vista, la teoría aristotélica de la suerte y la casualidad no viene a encarnar la resolución sistemática de un problema estrictamente filosófico, sino, antes bien, la respuesta y la contribución, desde la filosofía, a un interrogante insoluble anclado firmemente en la tradición literaria griega y en el cuestionamiento humano del lugar propio en el orden del mundo. Un interrogante que, pese a su extraordinaria complejidad, parece haber sido tradicionalmente abordado de tres únicas maneras: i) el azar no existe; ii) el azar es el origen de todas las cosas; iii) el azar es un instrumento al servicio de poderes divinos inescrutables para el intelecto humano. Aristóteles negará las tres opciones, tratando de disipar la confusión reinante en unas creencias comunes que asumen argumentos filosóficos irreconciliables entre sí o se amparan en viejas supersticiones. Como de costumbre, la respuesta de Aristóteles será compleja y mucho más precisa, pero no comenzará a perfilarse hasta las primeras líneas de Phys. II 5. 
1 Aristóteles, Phys. 196 a 12. La traduc-


pocas dificultades. Tanto en la lengua griega de Aristóteles como en el español contemporáneo a menudo empleamos de manera ambigua e intercambiable términos como "suerte", "coincidencia," "fortuna", "casualidad" o "azar". El empleo relajado y confuso que Aristóteles hace de ambos términos en Phys. II 4-5 se corresponde con su método de aproximación lingüística a la naturaleza de acontecimiento fortuito $y$, en realidad, no dista demasiado de nuestra propia experiencia lingüística de la incertidumbre. Si prestamos atención a nuestros usos cotidianos de expresiones relativas a la casualidad, la suerte y la fortuna constatamos la existencia de una terminología de la incertidumbre caracterizada por un grado de precisión ciertamente escaso, así como por una constante relajación e intercambiabilidad de los vocablos que la constituyen: azar, for tuna, casualidad, coincidencia, suerte, desgracia, accidente. Expresiones como "iqué casualidad!", “iqué suerte!" o "iqué coincidencia!" son empleadas indistintamente en marcos de aplicación sujetos a modificaciones constantes sin atender en absoluto a posibles diferencias internas que, sin duda, existen y reclaman un examen atento y una toma de distancia con respecto a la inercia que caracteriza el empleo cotidiano del lenguaje. Teniendo en cuenta estas consideraciones, y siempre que no recurra a una versión ajena, traduzco túxn por "suerte" y aútó $\mu \alpha \tau o v$ por "casualidad", reservando el término "azar" como concepto aglutinante tanto de los casos de suerte como de casualidad. Para el sentido preciso de la distinción aristotélica

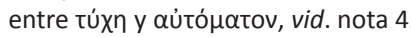

2 Temistio recurre al empleo cotidiano de la lengua griega a la hora de refutar a quienes niegan la existencia de túxn y aútó lópono se pregunta si dichos vocablos no constituyen meros nombres vacíos para ocultar la ignorancia humana en la atribución de las causas verdaderas que necesariamente subyacen a todo tipo de acontecimientos (In. Phys 188, 258 ss y 266). Por su parte, Simplicio considera la creencia común expresada en el lenguaje ordinario un auténtico regalo para la investigación filosófica, que deberá iniciar su andadura en contacto directo y crítico con los dones de la tradición transmitidos en el uso cotidiano del lenguaje (In Phys. 356-57).

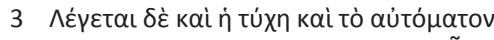

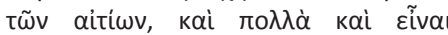

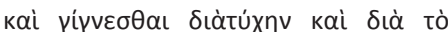

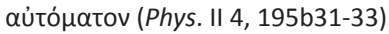

4 A lo largo de Phys. II 4-5, túxn y aútónatov son empleados de manera intercambiable y en clara oposición doctrinal con el contenido específico del capítulo 6, donde se introduce una distinción conceptual entre ambos vocablos que resulta fundamental tanto para la investigación natural como para la filosofía práctica del estagirita. En efecto, en 197â36-197b5 el ámbito de aplicación de túxn queda restringido a la esfera de los intereses humanos, mientras que aútó $\mu \alpha \tau o v$ se presenta como un concepto más amplio que aglutina todo acontecimiento excepcional, tanto en el orden natural como en el orden práctico. Asimismo, si acudimos a otros lugares del corpus observamos que en la mayor parte de ellos el empleo de los dos términos contradice las definiciones de II 6 , siendo túxๆ a menudo utilizada para cubrir tanto la dimensión de lo intencional como la de lo estrictamente natural, i.e, en el sen-

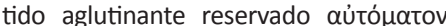
en II 6 (cf. Rh. I 5, 1361 b39-1362a12; I, 10-11, 1369a32-b5; EN III, 3, 1112 a 2133; An. Post | 30, 87b19-27; || 11, 94b2795a9; Protr. B 11; DC I 12, 283a29-283b3; GC, II, 6, 333b 3-7; Phys. II, 8198b30ss; PA I, 1640 a 27-33; Met. A, III, 984b 8-5; IX, 71048b37-1049a5). Dado que el interés del presente artículo se centra en Phys. II 4, asumo el empleo indiscriminado de ambos términos en dicho capítulo y eludo profundizar en el problema de sus matices diferenciales, para el cual pueden consultarse con provecho Phys. II 6, así como Mansion (1913, p. 324), Aubenque (1963, p. 90), Ross $(1975$, p. 355) y Dudley (1997, p. 94).

5 Para todas las referencias al texto español de Physica, sigo la traducción de G.R. Echandía (Aristóteles, 1995a).

6 Esto es, como causa material (tò દ̇\} oũ

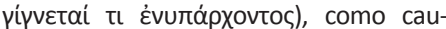

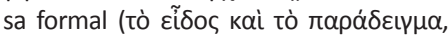



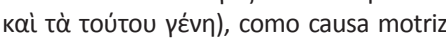

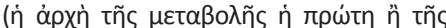

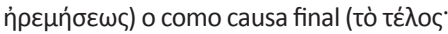

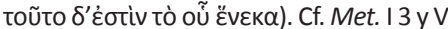
2, prácticamente idéntico a Phys. II 3.

7 En concreto, el primer interrogante quedará respondido en II 6, 1982-5, el segundo en II 6, 197a36-b37 y el tercero en
II 5, 196b10-197a35. Filópono (In Phys. 25920-24) nos recuerda que Aristóteles sigue en sus respuestas un orden inverso al que él mismo ha programado, respondiendo en primer lugar a la pregunta por

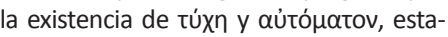
bleciendo después una distinción conceptual entre ambas nociones y, por último, investigando el tipo de causa en el que el azar en sentido amplio puede ser inscrito.

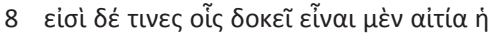

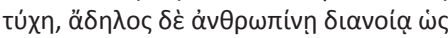

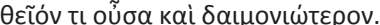

9 Una excelente exposición del problema y de sus conflictos internos puede consultarse en Rossi (2011, pp. 89-127 y, en especial, pp. 129-147)

10 “...quien, si bien empleó el azar en su кобцотоí $\alpha$, no permitió que fuera operativo en detalles tales como el hallazgo de un tesoro" (la traducción es mía).

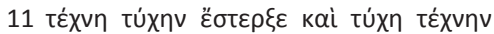
(EN114019-20)

12 “La expresión puede significar (1) 'el antiguo argumento' expuesto por los atomistas (como en S.330.14), o (2) 'el argumento mencionado con anterioridad'" (la traducción es mía).

13 Physica 254a16 y Politica 1282a15. El autor del De Mundo (401b25) cita el

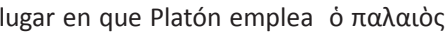

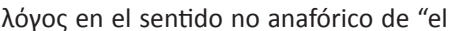
antiguo dicho", "el viejo refrán" (Leyes 715e8). Por lo demás, Platón se sirve de la misma expresión en otras dos ocasiones, Banquete 195b5 y Fedro 240 c1, ambas con un sentido no anafórico (para el sentido anafórico, vid. Phys. 254a16 y Pol. 1282 a 15). La interpretación de un sentido anafórico de la expresión ò $\pi \alpha \lambda \alpha$ เòs $\lambda$ ó Boeri (1993, p. 188), quien considera que "el antiguo argumento que niega el azar es el mencionado supra en 196a1-7 y que se remonta a los atomistas", así como por Norbert Kaul (1965, p. 19), que corrige la interpretación de Simplicio y favorece la de Ross. A diferencia de Ross y de Boeri, Kaul (ibid.) se niega a admitir la presencia de Demócrito en el primer grupo de opiniones, pues no alberga ninguna duda sobre la pertinencia de incluir al pensador atomista en el segundo y considera incongruente que Aristóteles divida específicamente las opiniones precedentes para hablar en el fondo de un solo grupo de pen- 
sadores. La interpretación de Kaul me parece sugerente. Sin embargo, el autor no ofrece ninguna respuesta a la pregunta que necesariamente se deriva de su propuesta: si Demócrito pertenece al segundo grupo de pensadores y no es posible que Aristóteles incluya a un mismo filósofo en dos grupos de opiniones distintos, ¿quiénes son los ह̌viot de 195b36?

14 In Phys. 330, 14-20. La traducción es mía.

15 El lector habrá observado que el primero de los dos ejemplos (el del encuentro casual de un tesoro al cavar un hoyo en la tierra con el fin de plantar un árbol) es utilizado por Aristóteles en Met. V, 30, 102515 y EN III, 1112a27. Alejandro de Afrodisia emplea un ejemplo similar en De anima libri mantissa (178, 1-5). En cuanto al segundo, E. Rohde ya señaló que se trata de una narración tan popular en la literatura antigua que no sería arriesgado afirmar que ella misma promoviera la leyenda de la muerte de Esquilo, quien, al parecer, murió descalabrado por una tortuga que un águila había dejado caer desde las alturas (Rohde, 1901).

16 La traducción es mía.

\section{Dante Alighieri (1989, canto IV, v. 136)}

18 Lamentablemente, no es este el lugar para una investigación pormenorizada al respecto. No obstante, cabe señalar que la mención del poeta florentino es mucho más que un capricho, una licencia poética o un comentario superficial. En realidad, la polémica contenida en sus palabras se extiende maravillosamente desde el pensamiento griego hasta la Divina Comedia, pasando por el comentario al Timeo de Proclo, las Instituciones Divinas de Lactancio, el De Natura Deorum de Cicerón o los comentarios de Tomás a las lecciones de filosofía natural (cf. Proclo. Comm in Timeo, 28 a; Lactancio, Inst. div. I, 2; Cicerón, De natura deorum I, 24, 66; Tusculanas, 11.22 y I, 18.42). Vincent Cioffari (1935, pp. 1-17) se ha ocupado in extenso de cubrir el debate sobre la convivencia del determinismo físico y el supuesto indeterminismo ontológico derivado de los fragmentos democríteos. A su juicio Dante -y junto a él Lactancio y Cicerón- estaban en lo cierto, pues la negación del carácter finalístico de la regularidad y el orden del cosmos no elimina el hecho de que Demócrito atribuya un origen objetivamente indeterminado al universo, esto es, un movimiento espontáneo o automático del cual se deriva una disposición regulada de la materia. Tal es, en efecto, la crítica feroz ejecutada por Aristóteles en Phys. II 4. Cioffari se propone mostrar que Dante no estaba equivocado por cuanto su opinión procedía, en efecto, del propio Aristóteles, que supo ver la contradicción de la propuesta democrítea mediante su concepto de causalidad indeterminada, por lo demás ajeno a las propuestas materialistas. Demócrito niega la finalidad y el azar en el mundo natural, diciendo que todo tiene una causa determinada y necesaria; afirma, en cambio, que el mundo y la esfera celeste se produjeron por torbellino y azarosamente, esto es, sin finalidad y, sobre todo, de modo indeterminado. Ese indeterminismo puede ser psicológico, en la medida en que la mente humana no capta la racionalidad de un proceso que objetivamente posee causas determinadas, o plenamente natural y objetivo. Aristóteles achaca a Demócrito un indeterminismo objetivo en el plano de lo celeste, lo cual es imposible desde el punto de vista de la armonía cósmica. Cioffari defiende que la crítica de Aristóteles no se limita a la negación de la teleología, sino a la afirmación de que el universo emerge espontáneamente $\mathrm{y}$, por tanto, de causas indeterminadas: mientras que dice que en el plano terrestre todo tiene una causa eficiente propia y definida, no especifica lo mismo en el caso de la regularidad celestial. Por ello habla de contradicciones internas en las teorías atomistas. Y, ciertamente, desde el enfoque aristotélico, dichas contradicciones existen.

19 Para la doctrina del vórtice ver Empédocles (DK 31 B 35) y Demócrito (DK 68 B 164 y 167).

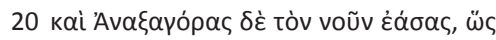

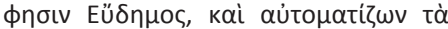

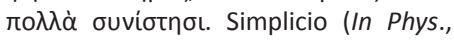
327, 26-27, la traducción es mía). Son conocidas las posiciones de Platón y Aristóteles con respecto a Anaxágoras en Fedón, 97bss y Met. I 4, 985a18, respectivamente.

21 Sobre la noción de túxn en Demócrito puede consultarse Bailey (1928), Blanche (1981), Buriks (1948), Cioffari
(1935, pp.1-16), Edmunds (1972), Langerbeck (1935), Vlastos (1945 y 1946).

22 Leunissen (2010, pp. 10-47).

23 Cf. Met. 1027a7ss, donde Aristóteles sostiene que la causa de aquello que llega a ser por accidente también es, por su parte, accidental.

24 А. каі $\Delta$ по́крเтоৎ каі оі $\Sigma \tau \omega$ เкоі

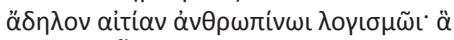

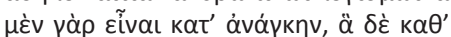

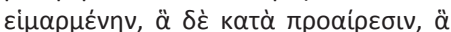

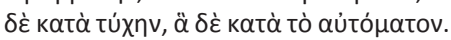
(DK 59 A 66) (La trad. es mía).

25 Uno de los primeros testimonios de este espíritu ilustrado lo encontramos en Jenófanes (DK 21 B 18), quien sostiene que los dioses no enseñaron a los hombres todas las cosas desde un comienzo, sino que fueron los propios mortales quienes, con el paso del tiempo, llegaron a los mejores descubrimientos sirviéndose de su propia inves-


en particular y el período ilustrado en general, véase el magnífico trabajo de Kube (1969). (Cf. Edelstein, 1967 e Isnardi Parente, 1966).

26 Sobre la relevancia del término ö $\delta \eta \lambda$ oc para una correcta interpretación de la ilustración ateniense en general, y de Anaxágoras en particular, vid. Diller (1932).

27 Orphei Hymni, 72.10 (Quand, 1962).

28 Verbeke (1961, p. 407) subraya las conexiones que vinculan el origen de toda reflexión filosófica con una pluralidad de contenidos tradicionales extraños a la prosa especulativa y que el discurso teórico, sin embargo, lejos de eliminar o asumir acríticamente, somete a un análisis exhaustivo orientado al esclarecimiento y la comprensión de los diversos interrogantes que asedian al ser humano. La filosofía, por tanto, se integra en una suerte de «alimentación de la investigación; esta permanece en contacto con la experiencia cotidiana, la experiencia de un mundo que se nos da a través de las opiniones comunes, las tradiciones, los mitos, la poesía, la religión, en un mundo que se nos ofrece con todos los aportes de la cultura humana» (la traducción es mía). (Cf. Nussbaum, 1986 , pp. $1-23$ y pp. $240-245)$. 


\section{BIBLIOGRAFÍA}

\section{Ediciones}

Aristóteles (1894). Ethica Nicomachea. Bywater, I. (ed.). Oxford: Clarendon Press.

Aristóteles (1955). De Caelo. Allan, D. J. (ed.). Oxford: Clarendon Press.

Aristóteles (1956). Physica. Ross, W. D. (ed.). Oxford: Clarendon Press.

Aristóteles (1957a). Metaphysica. Jaeger, W. (ed.). Oxford: Clarendon Press.

Aristóteles (1957b). Politica. Ross, W. D. (ed.). Oxford: Clarendon Press.

Aristóteles (1959). Ars rhetorica. Ross, W. D. (ed.). Oxford: Oxford University Press.

Aristóteles (1961). Aristotle's Protrepticus. An Attempt at Reconstruction. Düring I. (ed.). Göteborg-Stockholm: Almqvist $\&$ Wiksell.

Aristóteles (1968). Analytica Priora et Posteriora. Ross W. D. (ed.). London: Oxford University Press.

Aristóteles (1995a). Física. Madrid: Gredos.

Aristóteles (1995b). Ética a Nicómaco. Madrid: Gredos.

Arnim, H. von (ed.) (1964). Stoicorum Veterum Fragmenta. Stuttgart: Teubner.

Diels, H.; Kranz, W. (1951-1952). Die Fragmente der Vorsokratiker (6a ed.). Weidmann: Zürich-Hildesheim.

Juan Filópono (1887). In Aristotelis Physicorum, Libros Tres Priores. Vitelli, H. (ed.). Berlin: Reimer. http://dx.doi. org/10.1515/9783110807974

Simplicio (1882). In Aristotelis Physicorum, Libros Quattuor Priores. Diels, H. (ed.), Berlin: Reimer.

Temistio (1900). In Aristotelis Physica Paraphrasis. Shenkel, E. (ed.). Berlin: Reimer.

\section{Literatura secundaria}

Aubenque, P. (1963). La prudence chez Aristote. Paris: Presses Universitaires de France.

Bailey, C. (1928). The Greek atomists and Epicurus: a study. Oxford: Clarendon Press.
Blanche, L. (1981). Démocrite a-t-il cru au hasard? Revue de l'Enseignement philosophique, XXXI, pp. 27-33.

Boeri, M. D. (1993). Aristóteles. Física I y II. Boeri, M. D. (trad., introd. y com.). Buenos Aires: Biblos.

Buriks, A. A. (1948). Peri tuxhs. Leiden: Leiden University.

Cambiano, G. (1994). Automaton. Studi Storici. Rivista Trimestrale dell'Istituto Gramsci, 3, pp. 613-633.

Cioffari, V. (1935). Fortune and fate from Democritus to Sto. Tomas. New York: Columbia University.

Charlton, W. (1970). Aristotle's Physics. Books I and II. Oxford: Clarendon Press.

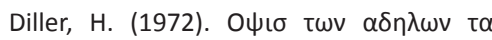
фaıvo $\mu \varepsilon v \alpha$. Hermes, 67, pp. 14-42.

Edmunds, L. (1972). Necessity, chance and freedom in the early atomists. Phoenix, 26, pp. 342-357. http://dx.doi. org/10.2307/1087594

Dudley, J. A. J. (1997). The evolution of the concept of chance in the Physics and Ethics of Aristotle. A commentary on Phys. II, 4-6. Leuven: Universiteit Nijmegen.

Edelstein, L. (1967). The idea of Progress in Classical Antiquity. Baltimore: John Hopkins University Press.

Isnardi Parente, M. (1966). Techne. Momenti del pensiero greco da Platone a Epicuro. Florencia: La Nouva Italia.

Kaul, N. (1965). Der Zufall und die Theorie des tragischen Handlungsablaufes bei Aristoteles. Köln: Universität zu Köln.

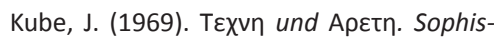
tisches und platonisches Tugendwissen. Frankfurt: Universtität Frankfurt am Main.

Langerbeck, H. (1935). Docis epirusmih. Studien zu Demokrits Ethik und Erkenntnislehre. Berlin: Neue Philologische Untersuchungen.

Leunissen, M. (2010). Explanation and teleology in Aristotle's sciencie of na- ture. New York: Cambridge University Press. http://dx.doi.org/10.1017/ CB09780511762499

Magris, A. (1984). L'idea di destino nel pensiero antico. Udine: del Bianco Editore.

Mansion, A. (1913). Introduction à la physique aristotélicienne. Lovaina-Paris: Institut Supérieur de Philosophie.

Micheli, G. (1998). II concetto di automa della cultura greca dalle origini al sec. IV a. C. Rivista della Storia della Filosofia, 3, pp. 421-462.

Nussbaum, M. C. (1986). The fragility of goodness. Luck and ethics in Greek tragedy and philosophy. Cambridge: Cambridge University Press.

Quand, W. (ed.) (1962). Orphei Hymni, Berlin.

Rohde, E. (1901). Der Tod des Aeschylus. En Kleine Schriften, vol. II. Tübingen: J.C.B. Mohr Verlag, pp. 209-211.

Ross, W. D. (1960). Aristotle's Physics. A revised text with introduction and commentary. Oxford: Clarendon Press.

Ross, W. D. (1975). Aristotle's Metaphysics. Oxford: Clarendon Press.

Rossi, G. (2011). El azar según Aristóteles: estructuras de la causalidad accidental en los procesos naturales y en la acción. Sainkt Agustin: Academia Verlag.

Verbeke, G. (1961). Philosophie et conceptions préphilosophiques chez Aristote. Revue Philosophique de Louvain, 59, pp. 405-430. http://dx.doi.org/10.3406/ phlou.1961.5083

Vlastos, G. (1945). Ethics and Physics? in Democritus (I). The Philosophical Review, 54, pp. 578-592. http://dx.doi. org/10.2307/2181547

Vlastos, G. (1946). Ethics and Physics? in Democritus (II). The Philosophical Review, 55, pp. 53-64. http://dx.doi. org/10.2307/2181570 